\title{
Therapy Adherence in Crohn's Disease: An Adult Case Study
}

\author{
Ronen Arai \\ Digestive Care of North Broward, Coral Springs, Fla., USA
}

\section{Key Words}

Crohn's disease · Diagnosis · Adherence · Serologic biomarkers · Case study

\begin{abstract}
Patients with Crohn's disease require long-term therapy and physician follow-up to treat symptoms, minimize complications of the disease, and address side effects that may occur due to therapeutic interventions. Once stabilized, it is not unusual for patients to question the need for continued therapy. This may be related to various factors including poor disease awareness, lack of adequate communication between the physician and the patient, not wanting to take medications chronically, and/or financial issues among a host of other possibilities. However, nonadherence can have a serious impact on morbidity and mortality in patients with Crohn's disease. This case illustrates the management and stabilization of a patient with active Crohn's disease and the use of diagnostic serological testing to aid in disease risk assessment and treatment guidance.
\end{abstract}

\section{Introduction}

In the absence of 'curative' treatment options, patients with Crohn's disease require continuous therapeutic management and monitoring by a physician [1]. Therapeutic choices depend on location of inflammatory lesions, severity of disease, and complications [1]. Treatment goals for Crohn's disease, like those for many other chronic diseases, include eradication of disease-related symptoms and restoration of the patient's quality of life while minimizing complications or therapy-related toxicities [1]. Success depends in part on patient adherence to the therapeutic regimen, which is influenced by many factors including the physician-patient relationship, disease awareness, effectiveness of the prescribed regimen, and adverse events associated with treatment.

Patients often doubt the severity of their Crohn's disease [2] and, therefore, empirical evidence of their disease and an objective measurement of disease severity can be a powerful tool to motivate adherence, especially after symptoms improve. The case presented here was chosen to illustrate the importance of ongoing therapy to a positive outcome based on objective diagnostic findings and patient education. 


\section{Case Report}

A 21-year-old man was admitted to the emergency department of a hospital in December 2007 complaining of nausea/vomiting and periumbilical pain. Computed tomography of the abdomen/pelvis revealed ileal thickening and a small bowel obstruction. The differential diagnosis was infectious enteritis versus Crohn's disease. The patient was admitted to the hospital and treated with nasogastric suction, intravenous fluids, and antibiotics. He subsequently improved and was discharged.

Soon after, the patient presented at our office with complaints of recurrent abdominal pain, new-onset diarrhea, and rectal bleeding. A colonoscopy in April 2008 demonstrated ulcerations of the ileum and colon consistent with Crohn's disease; the ileum was notably stenotic, and only the distal few centimeters could be visualized (fig. 1). A small bowel follow-through study with barium contrast showed the stenoses and ulcerations extending over the distal $30 \mathrm{~cm}$ of the ileum and confirmed a diagnosis of Crohn's disease. Treatment with mesalamine $4 \mathrm{~g}$ /day and a 14-day course of metronidazole relieved the abdominal pain and bleeding, but the diarrhea persisted.

Over the next 2 weeks, the patient developed epigastric pain and nausea and was admitted to the hospital for severe pain. A diagnosis of pancreatitis likely due to the mesalamine prompted discontinuation of the drug and initiation of budesonide $9 \mathrm{mg}$ /day. Symptoms of pancreatitis subsequently improved, and the patient was discharged from the hospital after a short stay. Over the next 3 weeks, the diarrhea, bleeding, and pain resolved.

However, by the end of August 2008, the diarrhea and cramping periumbilical pain recurred, and an immunomodulator was added to the therapeutic regimen. Ten days later, the patient was admitted to the hospital with a high fever $\left(103^{\circ} \mathrm{F}\right)$ associated with elevated liver enzymes and lymphopenia. Workup for bacterial infection was negative, and a viral illness was presumed. The immunomodulator was discontinued as it was presumed that the lymphopenia had made the patient susceptible to the infection.

In November 2008, at the follow-up appointment in the physician's office, a lengthy discussion with the patient included the care of Crohn's disease and the risks and benefits of biologic therapy. A decision was made to initiate induction therapy with the anti-tumor necrosis factor (TNF) agent adalimumab $160 \mathrm{mg}$ s.c. then $80 \mathrm{mg}$ s.c. 2 weeks later, followed by maintenance therapy at a dose of $40 \mathrm{mg}$ s.c. every 2 weeks. By March 2009, the patient was in clinical remission and had regained $15 \mathrm{lb}$. A follow-up colonoscopy demonstrated complete healing of the colonic mucosa and significant ileal healing as well (fig. 2).

At this point, the patient questioned the need for long-term treatment with adalimumab. He preferred to stop the medication because he was feeling well and because he was concerned about the potential for adverse effects. A Prometheus IBD Serology 7 test was ordered to help assess the need for ongoing therapy based on serologic markers. Results revealed elevations of the following biomarkers for Crohn's disease: ASCA (both IgA and IgG), anti-OmpC IgA, anti-CBir1, and inflammatory bowel disease (IBD)-specific pANCA (table 1). After additional discussion about the prognostic value of elevations of multiple markers and the high risk for disease progression, the patient agreed that continued anti-TNF therapy was indicated. The patient continues to feel well without adverse effects of therapy.

\section{Discussion}

Several studies have examined nonadherence to treatment for IBD. In one such prospective study, Cerveny et al. [2] found that $38.9 \%$ of patients were intentionally nonadherent to their IBD regimen during the 2 weeks prior to being interviewed and that patients' disease awareness and medication knowledge are important influences on adherence.

Serologic testing such as with the Prometheus IBD Serology 7 panel has evolved over time - from its initial use in helping differentiate ulcerative colitis from Crohn's disease to further evaluating indeterminate colitis, stratifying disease, predicting the potential course of IBD, determining more severe disease phenotypes, and guiding treatment based on the prognosis of the patient. Serologic testing can determine the level of immune reactivity to various markers of disease and, therefore, can be used to predict an increased risk of 
complicated disease requiring ongoing treatment [3]. The serologic results for our 21-year-old patient were communicated clearly to him, improving his disease awareness and ability to make informed decisions during treatment. The results facilitated an extensive discussion that strengthened his knowledge of the medication and increased his appreciation of the importance of adherence.

Mow et al. [4] were the first investigative group to associate increased levels of immune response with complications of Crohn's disease. Specifically, these investigators showed that specific serologic markers are present in patients with a more aggressive disease phenotype, in whom both the number and level of antibody responses were significantly associated with an increased likelihood of fibrostenosis, internal perforating disease, and the need for small bowel surgery. Arnott et al. [5] also found an association between immune responses to ASCA, anti-I2, anti-OmpC, and pANCA in patients with a more severe Crohn's disease phenotype that included small bowel disease, longer duration of disease, a higher risk for progression, and the need for intestinal surgery. Similar correlations between the number and level of immune response and more severe disease phenotype have been observed in the pediatric population as well $[6,7]$.

The IBD Serology 7 results obtained in this case served as a valuable tool for overall disease management and facilitated physician-patient communication on disease prognosis and a continued need for therapy. The results yielded useful data to help ensure duration of therapy and adherence.

\section{Acknowledgment}

Editorial support was provided with funding by Prometheus Laboratories Inc.

\section{Conflict of Interest Disclosure}

Ronen Arai is a speaker for Prometheus Laboratories Inc.

Table 1. Levels of serum biomarkers and reference values in a patient diagnosed with IBD and, specifically, Crohn's disease as measured by enzyme-linked immunosorbent assay

\begin{tabular}{lcl}
\hline Assay $(\mathrm{EU} / \mathrm{ml})$ & Patient & Reference \\
\hline ASCA IgA & 32.7 & $<20.0$ \\
ASCA IgG & 95.6 & $<40.0$ \\
Anti-OmpC IgA & 16.7 & $<16.5$ \\
Anti-CBir1 & 108.4 & $<21.0$ \\
IBD-specific pANCA & & $<12.1$ \\
$\quad$ Autoantibody & 49.5 & not detected \\
$\quad$ Perinuclear pattern* & detected & not detected \\
\hline
\end{tabular}

* Measured by immunofluorescence assay. ASCA IgA and IgG = Anti-Saccharomyces cerevisiae antibody immunoglobulin A and G; anti-OmpC = antibodies to outer membrane porin C of Escherichia coli; anti-CBir 1 = antibody against CBir 1; pANCA = perinuclear antineutrophil cytoplasmic antibody. 


\begin{tabular}{r|l|l|l}
$\begin{array}{r}\text { Case Reports in } \\
\text { Gastroenterology }\end{array}$ & $\begin{array}{l}\text { Case Rep Gastroenterol 2010;4:153-157 } \\
\text { D0I: 10.1159/000314230 }\end{array}$ & Published online: May 12, 2010 & $\begin{array}{l}\text { @ 2010 S. Karger AG, Basel } \\
\text { ISSN 1662-0631 } \\
\text { www.karger.com/crg }\end{array}$ \\
\hline
\end{tabular}

Fig. 1. A colonoscopy in November 2008 demonstrated ulcerations of the ileum and colon consistent with Crohn's disease. A stenotic ileum allowed only the distal few centimeters to be viewed. The physician initiated induction therapy with adalimumab $160 \mathrm{mg}$ s.c., then $80 \mathrm{mg}$ s.c. 2 weeks later, followed by maintenance therapy at a dose of $40 \mathrm{mg}$ s.c. every 2 weeks.
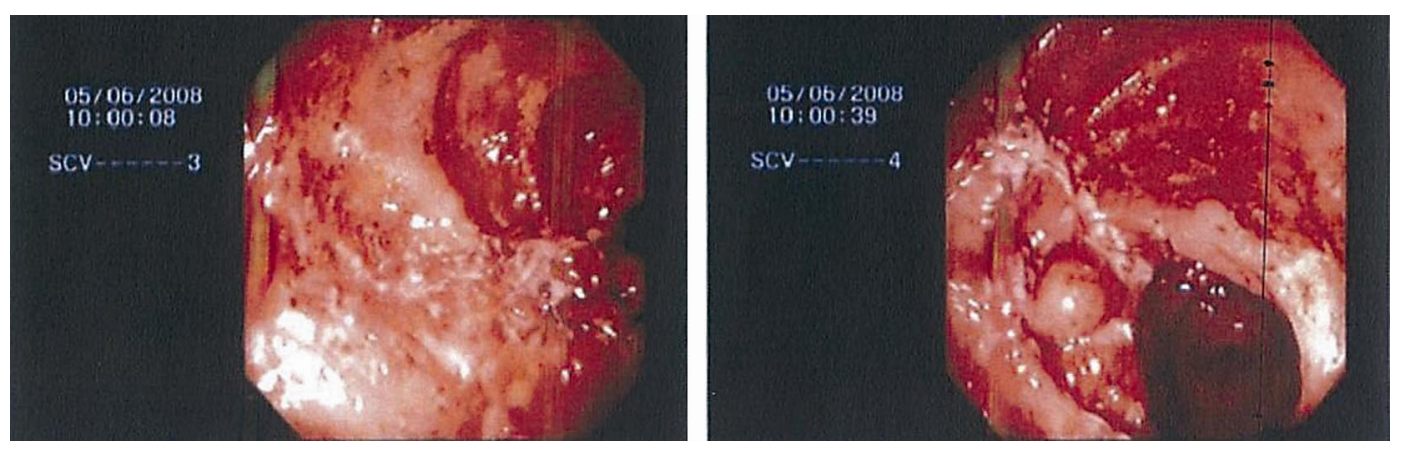

Fig. 2. A follow-up colonoscopy in March 2009 after a regimen of adalimumab demonstrated healing of the colonic mucosa and ileum. An inflammatory polyp at the ileocecal valve was removed.
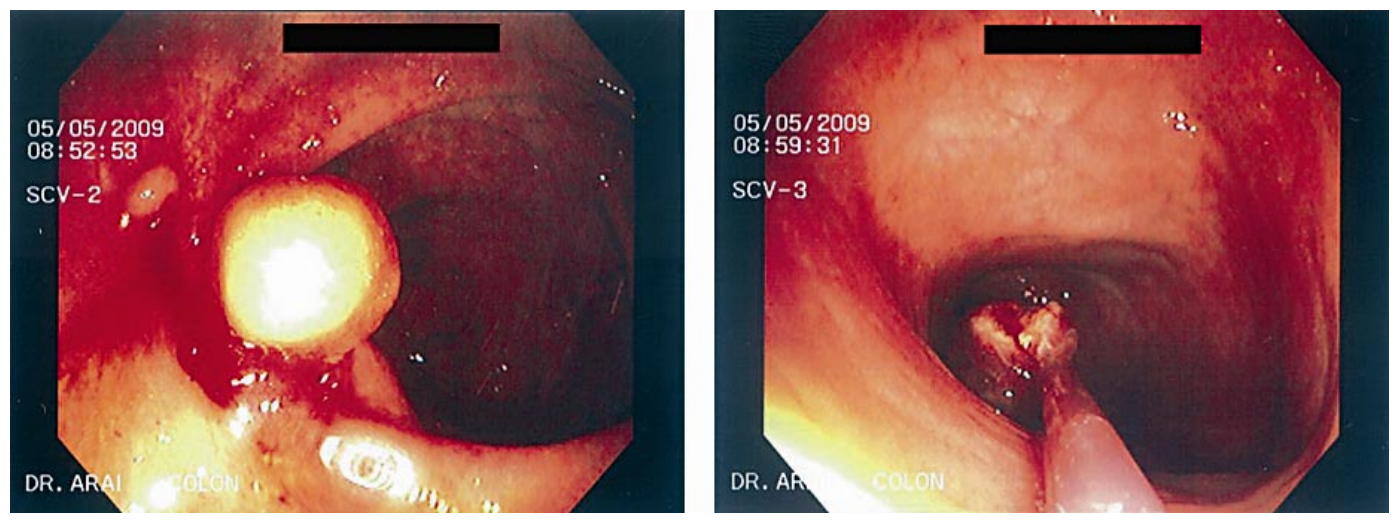


\section{References}

-1 Lichtenstein GR, Hanauer SB, Sandborn WJ; the Practice Parameters Committee of the American College of Gastroenterology: Management of Crohn's disease in adults. Am J Gastroenterol 2009;104:465-483.

-2 Cerveny P, Bortlik M, Kubena A, Vlcek J, Lakatos PL, Lukas M: Nonadherence in inflammatory bowel disease: results of factor analysis. Inflamm Bowel Dis 2007;13:1244-1249.

3 Dubinsky MC, Hanauer SB: Emerging strategies in the use of IBD-related serologic markers. Gastroenterol Hepatol 2008;4:1-3.

-4 Mow WS, Vasiliauskas EA, Lin YC, Fleshner PR, Papadakis KA, Taylor KD, Landers CJ, Breu-Martin MT, Rotter JI, Yang H, Targan SR: Association of antibody responses to microbial antigens and complications of small bowel Crohn's disease. Gastroenterology 2004;126:414-424.

- 5 Arnott ID, Landers CJ, Nimmo EJ, Drummond HE, Smith BK, Targan SR, Satsangi J: Sero-reactivity to microbial components in Crohn's disease is associated with disease severity and progression, but not NOD2/CARD15 genotype. Am J Gastroenterol 2004;99:2376-2384.

-6 Dubinsky MC, Lin YC, Dutridge D, Picornell Y, Landers CJ, Farrior S, Wrobel I, Quiros A, Vasiliauskas EA, Grill B, Israel D, Bahar R, Christie D, Wahbeh G, Silber G, Dallazadeh S, Shah P, Thomas D, Kelts D, Hershberg RM, Elson CO, Targan SR, Taylor KD, Rotter JI, Yang H: Serum immune responses predict rapid disease progression among children with Crohn's disease: immune responses predict disease progression. Am J Gastroenterol 2006;101:360-367.

-7 Dubinsky MC, Kugathasan S, Mei L, Picornell Y, Nebel J, Wrobel I, Quiros A, Silber G, Wahbeh G, Katzir L, Vasiliauskas E, Bahar R, Otley A, Mack D, Evans J, Rosh J, Hemker MO, Leleiko N, Crandall W, Langton C, Landers C, Taylor KD, Targan SR, Rotter JI, Markowitz J, Hyams J: Increased immune reactivity predicts aggressive complicating Crohn's disease in children. Clin Gastroenterol Hepatol 2008;6:1105-1111.

Abbreviations: anti-CBir1 = antibody against CBir1; anti-I2 = antibodies against Pseudomonas fluorescens; anti-OmpC = antibodies against outer membrane porin C of Escherichia coli; ASCA IgA and IgG = anti-Saccharomyces cerevisiae antibody immunoglobulin A and G; pANCA = perinuclear antineutrophil cytoplasmic antibody. 